\title{
Designing Robust Tablet Formulas Resilient to Raw Material and Equipment Variations with the Aid of Design of Experiment (DOE) Method
}

\author{
Tian Hao*, Denise Doerflein Fulk \\ Department of Science and Technology, Pharmavite LLC, Valencia, USA
}

Email address:

thao@pharmavite.net (Tian Hao)

${ }^{*}$ Corresponding author

\section{To cite this article:}

Tian Hao, Denise Doerflein Fulk. Designing Robust Tablet Formulas Resilient to Raw Material and Equipment Variations with the Aid of Design of Experiment (DOE) Method. International Journal of Nutrition and Food Sciences. Vol. 8, No. 6, 2019, pp. 78-85.

doi: $10.11648 /$ j.ijnfs.20190806.11

Received: June 18, 2019; Accepted: December 6, 2019; Published: December 12, 2019

\begin{abstract}
In tablet production, the physical properties of tablet products are found to change from batch to batch occasionally, which could be caused by raw material and equipment variations. This article focuses on how to design robust formulas resilient to raw material and equipment variations with the aid of Design of Experiment (DOE) method, how to optimize the concentrations of each individual ingredient, and how to avoid the common production issues related to tablet capping, chipping, and dusting problems. The impacts of particle sizes and equipment on compression behaviors were experimentally evaluated and explained with a theoretical hypothesis. The main goal is to show how tablet product quality is controlled from fundamental scientific principles when many active ingredients are present in a single formula, hard to be engineered and dramatically different from the pharmaceutical products. Our findings may provide insightful clues on tablet formulations and design strategies.
\end{abstract}

Keywords: Tablets, DOE (Design of Experiment), Raw Materials, Capping, Chipping

\section{Introduction}

In typical tablet formulas, there should be a lubricant that helps tablets eject from the die, a powder flow agent that helps the powder blend flow from the hopper to the die area, a disintegrant that helps the tablets dissolve in a medium or inside the human body, a binder that binds all particles together to reach the desired hardness and friability, fillers that help achieve the desired size, and active ingredients that provide nutritional and therapeutic benefits [1-3]. In contrast to the pharmaceutical formulas that usually contain one active ingredient, nutraceutical formulas often contain more than one active ingredient, sometimes more than 10 , making nutraceutical formulations challenging in terms of how to design robust formulas resilient to raw material and equipment variations.

There are many factors that contribute to common tablet problems during and after productions, most of which are related to formula design, tooling and compression variations, and environmental condition fluctuations [4-9]. At formula design stage, critical attributes need to be identified with the least amount of experiment runs to allow for short formula development time. The formulas related critical issues include raw material particle size variation, excessive amount of lubricants, insufficient amount of binder, unoptimized concentration of ingredients, and non-uniformity or segregation in the powder blend. The tooling and compression related issues include punch dwell time differences among compression equipment during scale-up and manufacturing, poor tooling design, and compression force variations. The environmental conditions, such as the room humidity and temperature during raw material storage and production of final products, affect particle surface moisture and impact the physical properties of the final tablet. These factors can be critical to the integrity of the tablet, and the most critical ones may depend on each formula [4-9].

With many different ingredients in nutraceutical formulas and a broad spectrum of process factors, a statistical tool like 
the Design of Experiment (DOE) [10] can be employed to efficiently identify the critical attributes and optimize the concentration of each ingredient. DOE method allows for changes in multiple parameters simultaneously, enable quick understanding of the impact of each factor and the potential interactions between factors, and have the ability to identify a raw material with significant impact on final product properties and determine optimum concentration. Utilizing a DOE method can substantially reduce the number of experiments and expedite the formulation development process. This article aims to demonstrate how a DOE method can be used to optimize tablet formulas to withstand the raw material and production variations, and ultimately minimize production failures.

\section{Experiment Methods and DOE}

Several studies are presented to demonstrate different applications of DOE through the product development and production processes. Experimental procedures and DOE methods used are introduced below.

\subsection{Methods}

The tablet formulas were weighed and blended with lab- scale instruments and equipment. The ingredients required for a typical nutraceutical tablet formulation are listed in Table 1 with the functionalities and typical amounts. The concentrations of ingredients were varied in these studies intentionally to gauge the impacts on the physical properties of the tablet and associated powder blend, including blend flowability, tablet hardness, friability, disintegration time, and chipping and capping points.

The particle size of powders were measured either with the standard sieving analysis method using Ro-tap equipment or Horiba laser diffraction instrument LA-950. For separating a large quantity of powder blends of varying particle size, Ro-tap sieve shaker with mesh sizes ranging from 20 to 200 meshes were used. For evaluating powder flowability, a Flodex or a glass funnel was used based on the orifice size: i.e., A powder that can flow through a smaller orifice is considered to have a better flowability than the one that flows through a larger orifice.

Two types of tablet press were used: a single punch Carver press and 27 station IPR Unipress. The compression profile expressed as hardness against compression force, was generated from both presses for assessing the scalability of the formulas. For compression profiles shown in this article, each data point is the average of at least five tablets.

Table 1. Typical nutracentical formulas.

\begin{tabular}{lll}
\hline Materials & Concentration & Functionality \\
\hline Active ingredients & Varied & Major ingredient(s), typically ranging from 1-10. \\
Flow agent $\left(\mathrm{SiO}_{2}\right)$ & $0.5 \sim 2 \%$ & Flow or anti-caking agent \\
Lubricant $(\mathrm{MgSt})$ & $0.5 \sim 2 \%$ & Lubrication \\
Fillers & $10 \sim 70 \%$ & Bulking agent and potentially flow aid \\
Binder & $5 \sim 25 \%$ & Bind particles together \\
Flavors & $0.2 \sim 1 \%$ & Taste modifier, optional \\
Colorant & $0.5 \sim 3 \%$ & Coloring tablets, optional \\
\hline
\end{tabular}

\subsection{DOE}

The DOE was created and analyzed with Minitab software. For reducing the number of runs, a half factorial design was implemented. Typically, a low and high concentration of each ingredient are chosen and a center point is included in the design. For identifying critical ingredients, we chose 4 or 5 raw materials, one or two in each category shown in Table 1, for DOE. For the formulas containing more than one filler, the fillers of the highest concentrations would be used. Data analysis was performed with Minitab software, which utilized the prediction tool embedded in the software. A confirmation run was performed to check for accuracy of the DOE. An example of a DOE design space is shown in Figure 1, where four raw materials were chosen: granular mannitol (GM) with a concentration range 20-70 $\mathrm{wt} \%$, magnesium stearate $(\mathrm{MgSt})$ with a concentration range 0.5-1.5 wt $\%$, silicon dioxide $\left(\mathrm{SiO}_{2}\right)$ with a concentration range $0.25-1 \mathrm{wt} \%$, steric acid (SA) with a concentration range $0-2 \mathrm{wt} \%$, and a center point for each raw material. The hardness, friability, flowability of formula blend, and disintegration time were chosen as responses.



Figure 1. An example of DOE design space.

\section{Results and Discussions}

Experimental results and discussions are presented in this section and focuses are placed on identification of critical attributes, impact of the particle sizes of whole blends and binders, and a physical mechanism hypothesis to explain the data. Many experiments were executed and the data presented are extracted from the collective studies to demonstrate the methodology. 


\subsection{Identification of Critical Attributes and Component Levels}

DOE method was employed to identify statistically significant attributes in the formulas based on critical physical properties of the tablet. Three physical parameters of tablets (hardness, friability, and disintegration time) were chosen in response to the design to address chipping and capping issues. Chipping or capping could be caused by under-compressed tablets or by overly compressed tablets. When particles are under-compressed, the applied compression force is not strong enough to overcome interfacial energy for creating the bonds between particles; When particles are overly compressed, particles inside tablets have been mechanically crushed, thus the structure of each particle is weakened even destroyed and particles are merely weakly connected, unable to hold the tablet intact. The flowability of formula blends was also evaluated as a design response, as this is a critical attribute to the manufacturability of tablet formula.

To demonstrate how to identify critical attributes and optimize component levels impacting tablet properties, a study investigating a product that exhibited chipping and capping issues is showed. For avoiding of re-registering formulas with government agencies, we were limited to make changes to the ingredients in the formula, except the ratios among the ingredients within a certain acceptable range. Ingredients significantly impacting hardness of the tablet using DOE method were identified for potentially resolving compression issues. The hardness at the compression force $12 \mathrm{kN}$ was used to assess the compressibility, and a total of 9 formulas were prepared and compressed. Factor B, the granular mannitol, was statistically significant with the alpha parameter $\leq 0.05$ (Figure 2). With increasing concentration of granular mannitol from $20 \%$ to $70 \%$, tablet hardness increases from 10 to $30 \mathrm{scu}$ (Strong-Cobb Unit, $1 \mathrm{scu}=7.005$ Newtons), as shown in Figure 3. Note that the scattering of data points at $20 \%$ and $70 \%$ of granular mannitol was expected, as the concentrations of other ingredients differed between each experiment.



Figure 2. The probability vs. effect for identifying the statistically significant attributes in the formula. Only one factor, granular mannitol, is statistically significant with the alpha parameter $\leqslant 0.05$.

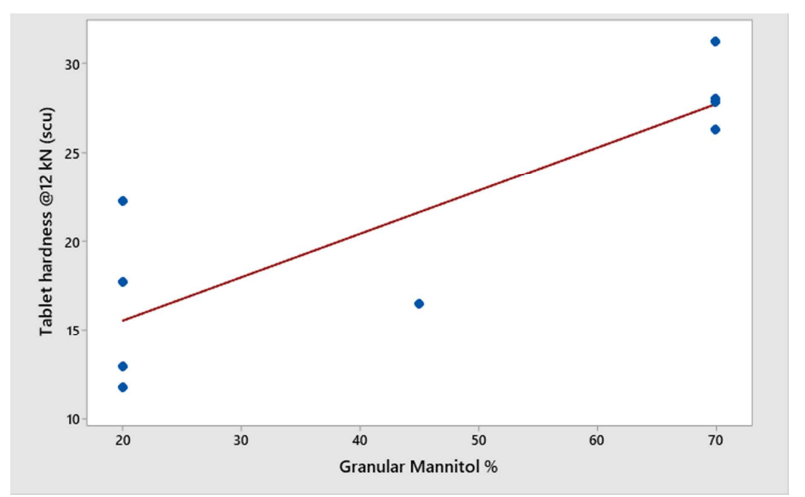

Figure 3. Hardness under $12 \mathrm{kN}$ compression force vs. concentration of granular mannitol.

Similar data analyses were performed for flowability and disintegration time to ensure that flowability and disintegration time are not affected by concentration adjustments. This practice would shed light on what is the safe operating space for the formula during production. The concentrations of major ingredients can vary safely without generating unexpected changes to blend or tablet physical properties, which could result in defects during the manufacturing process. There was no statistically significant effect observed for disintegration time (Figure 4) and flowability (Figure 5), when the concentrations of silicon dioxide ( $\mathrm{SiO} 2$ ), granular mannitol, magnesium stearate (MgSt), and stearic acid (SA) are adjusted, indicating a broad range of flexibility for the studied formula with respect to disintegration time and flowability. From Figures 3 to 5 , we can conclude that any concentrations within the respective ranges of studied ingredients of silicon dioxide, granular mannitol, magnesium stearate, and stearic acid can be used in this formulation without jeopardizing powder blend flowability and tablet disintegration time. The impact of each component level on tablet hardness at $12 \mathrm{kN}$ compression force was evaluated (Figure 6). For both silicon dioxide and magnesium stearate, the hardness decreased at the mid-point of concentration range evaluated, indicating that either of the extreme conditions can be chosen to achieve the highest hardness. For granular mannitol, the hardness didn't increase with the concentration until it reached above $45 \%$; at lower concentrations, there was minimal effect of concentration on hardness. The impact of stearic acid negatively effected hardness (reduced hardness). Stearic acid is a highly hydrophobic material, easily sticking on the metal surfaces and forming aggregates and lumps inside the powder bed, potentially contributing to chipping and capping issues. Such aggregates were observed in these formula blends even after the sieving and blending processes. Although it has a somewhat binding capability due to its long fatty acid chain, stearic acid is generally considered a secondary lubricant to magnesium stearate and less widely used in tablet formulas due to its incompatibility with other ingredients [11]. Based on the stearic acid concentration impact on tablet hardness and the observed lumps in the powder blend, we removed stearic acid from the formula to maximize the compressibility. 


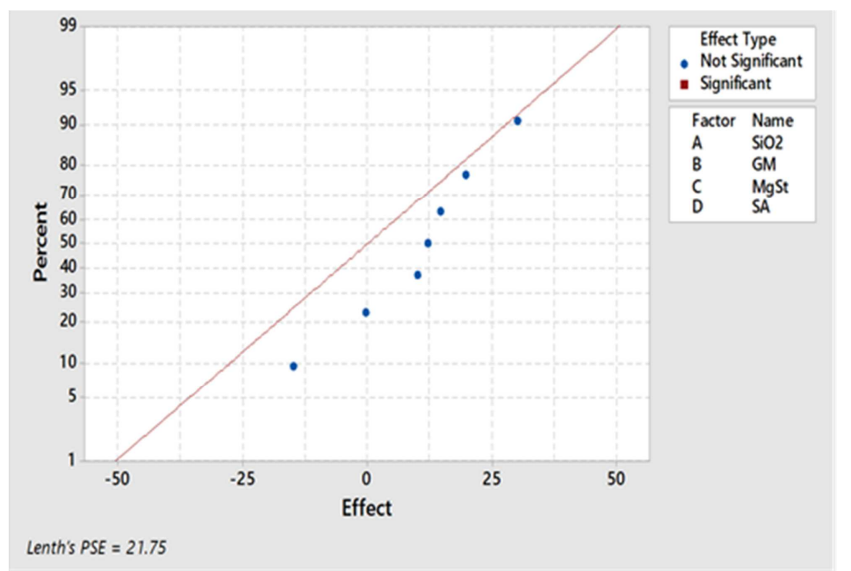

Figure 4. Probability vs. effect for identifying the statistically significant attributes in the formula for the disintegration time.

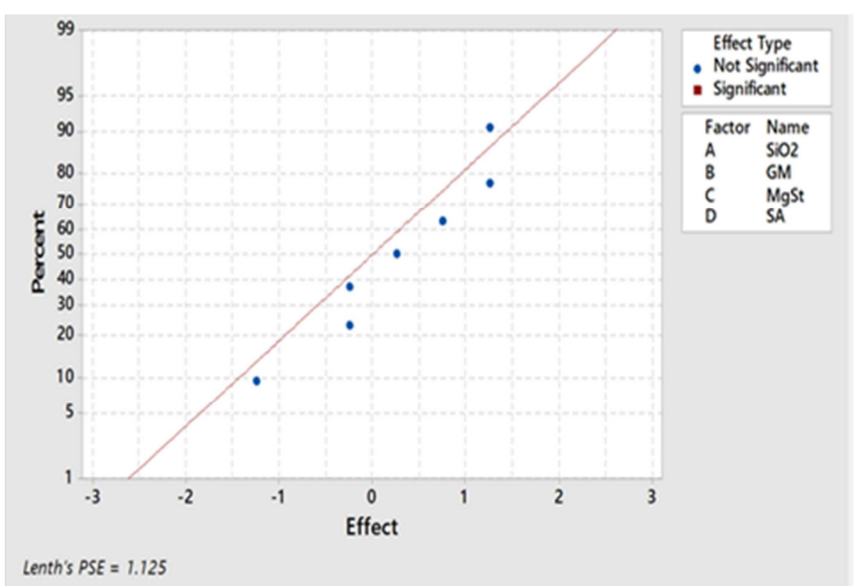

Figure 5. Probability vs. effect for identifying the statistically significant attributes in the formula to the flowability.

The DOE analysis identified granular mannitol as a critical ingredient that should have a concentration more than $45 \%$, and revealed the optimal concentrations of other ingredients like silicon dioxide, magnesium stearate, and stearic acid to achieve maximum hardness. The optimized formula contained $0.25 \%$ silicon dioxide, $1 \%$ magnesium stearate, $60 \%$ granular mannitol, and $0.5 \%$ stearic acid; it showed improved compressibility, but still had chipping issues at high compression forces. Besides the concentrations, the particle sizes of each ingredient and binder material in the formula can impact physical properties of tablets, which will be evaluated in the next sections.

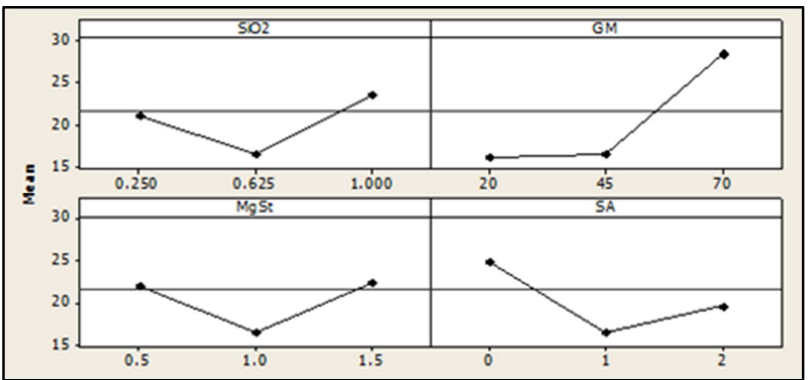

Figure 6. The main effect plot of the mean hardness vs. the concentrations of each ingredient.

\subsection{Particle Size Impact}

For powder form ingredients, the most common variation is particle size, as controlling particle size distribution range is not easy during production even with well-controlled processing conditions. A robust formula must be designed to be resilient to particle size variations, therefore the physical properties of tablets can be kept consistent from batch to batch within the acceptable ranges. Particles size distributions of three different lots of dextrose measured with the Horiba particle sizer are depicted in Figure 7. Two lots had nearly identical unimodal particle size distributions, but the third had a bimodal distribution, dramatically different from the other two. This type of variation could result from the production process itself, particle segregation during shipment and storage, or improper discharging technique. Dextrose is a small-molecule material that can be manufactured consistently with well-defined processes. Raw materials with relatively large molecules may be harder to manufacture and particle size variation could be even more pronounced. Botanical extracts are an example of such challenging materials; bimodal and trimodal particle size distributions with a very wide range from a few microns to a few millimeters are frequently observed. Wide particle size distribution variations could impose a huge impact on compression profiles.



Figure 7. The particle size distribution of three different lots of dextrose, a well-controlled product.

To address the particle size impact on compressibility, we intentionally separated a complete tablet blend into three fractions of different particle size ranges using Ro-tap equipment. While this approach of sieving an entire tablet blend is not practically employed during tablet manufacture and may result in some composition differences among the sieve fractions collected, it was used to quickly understand the impact of particle size by creating drastic changes to the overall blend. The compression profiles of these three fractions are shown in Figure 8. For the high and medium particle size ranges, the compression profiles are very similar within the compression force range 5 to $35 \mathrm{kN}$. These results indicate that the compression profile may not change if the particle size of the whole formula is above 170 microns. However, once the particle size is below 149 microns, the 
blend becomes less compressible and requires greater compression force to achieve the same harness compared to the blends produced with larger particles. Figure 8 demonstrates that particle size variation could potentially shift the compression profile, resulting in unexpected changes to tablet properties.



Figure 8. The compression profiles of the same formula blend sieved into three fractions of different particle size ranges.

To ensure consistent compressibility of tablet formulations, particle size of all ingredients should be controlled in an acceptable range and an adequate amount of a binder material like microcrystalline cellulose (MCC) may be added to compensate for variation of raw materials. MCC is available in many grades with different physical properties. Three grades of MCC with different particle sizes, bulk densities, and morphologies from Asahi Kasei were evaluated and their physical characterization is shown in Table 2. UF-711 and KG-1000 grade MCCs have a similar particle size about $50 \mu \mathrm{m}$, but they have different bulk densities and particle morphologies. KG-1000 are needle-shaped particles and expected to show a relatively poor flowability in comparison with UF-711, which is more spherical with a porous structure. $\mathrm{PH}-102$ has a relatively large particle size of about $90 \mu \mathrm{m}$ and the highest bulk density. The particles of PH-102 are spherical and hard, which may contribute to higher bulk density. Each grade was evaluated with the same concentration of $\mathrm{MCC}$ in a tablet formulation. The compression profiles of the formulas are shown in Figure 9. The formula with UF-711 MCC is the most compressible, followed by KG-1000 and $\mathrm{PH}-102$, respectively. The compressibility differences are induced by the particle size and particle morphology differences among the grades of MCCs. PH-102 has been widely used in various formulas due to its lower cost and good flowability; however, its capability for enhancing compressibility is limited in comparison with the smaller particle sized MCC. Although both UF-711 and KG-1000 are more compressible than PH-102, the compressibility improvement observed with $\mathrm{PH}-102$ was sufficient in this formulation and thus it was chosen for further evaluation of MCC concentration impact on compressibility. The compression profiles generated with the Carver press are shown in Figure 10, including the original formula, the optimized formula described in section 3.1, and the further enhanced formulas with $10 \mathrm{wt} \%$ and $20 \mathrm{wt} \%$ PH-102 MCC. The optimized formula is very compressible in comparison with the original formula, and they contain the same ingredients with slightly different concentrations. The compressibility was further improved with the addition of MCC to the optimized formula. The compression data demonstrate the power of the optimization process through DOE, as compressibility was dramatically improved without adding new ingredients. Addition of MCC as a dry binder resulted in even further improvements to the product.

Table 2. Microcrystalline Cellulose (MCC) used in the study [12].

\begin{tabular}{llll}
\hline Grade & $\begin{array}{l}\text { Average particle } \\
\text { size }(\boldsymbol{\mu m})\end{array}$ & $\begin{array}{l}\text { Bulk density } \\
\left(\mathbf{g} / \mathbf{c m}^{\mathbf{3}}\right)\end{array}$ & $\begin{array}{l}\text { Particle } \\
\text { morphology }\end{array}$ \\
\hline UF-711 & 50 & 0.22 & $\begin{array}{l}\text { Porous, } \\
\text { spherical }\end{array}$ \\
KG-1000 & 50 & 0.12 & $\begin{array}{l}\text { Needle-shaped } \\
\text { PH-102 }\end{array}$ \\
\hline
\end{tabular}

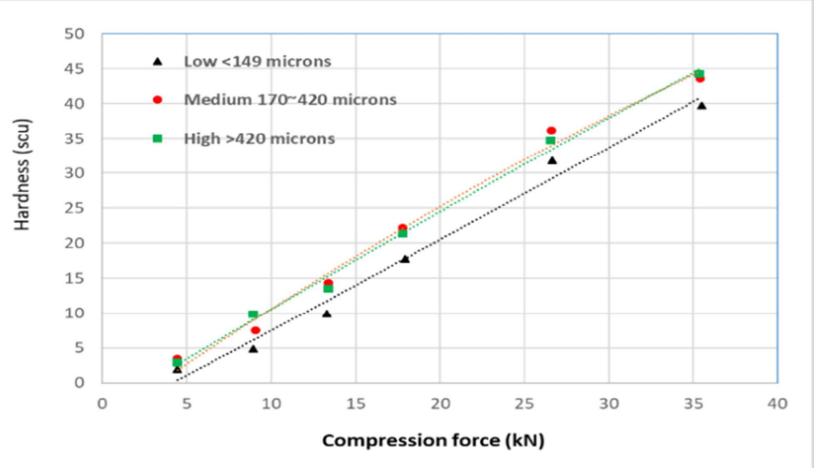

Figure 9. The compression profiles of the same formula but containing different grades of microcrystalline celluloses (MCC) from Asahi Kasei.



Figure 10. The compression profiles of the original formula, the optimized formula, $10 \mathrm{wt} \%$ MCC and $20 \mathrm{wt} \%$ MCC PH-102 formulas obtained with the single punch Carver press.

The binder particle size, morphology, and the concentration are all critical to the compression profile. The trend of the experimental data obtained at the smallest scale with the Carver press was confirmed with a 27- station automatic IPR press and the results are shown in Figure 11. The compression profile improvements are preserved among these formulas, with the order expected: the formula with 20 $\mathrm{wt} \%$ MCC provided the best performance, followed by the 
optimized formula and the original formula. Most importantly, chipping and capping issues are only observed at the highest compression force for both the original and optimized formulas, $21 \mathrm{kN}$ and $18 \mathrm{kN}$, respectively, as shown in Figure 11. Since the optimized formula can achieve the same hardness at lower compression forces, such chipping and capping issues can be avoided. There is no chipping or capping issue observed with the formula of $20 \mathrm{wt} \% \mathrm{PH}-102$ over the entire compression force range. Both the optimized and $20 \mathrm{wt} \% \mathrm{MCC}$ formulas show a much wider operating range for production. When there was variation in equipment or ingredient particle size, these formulas can be compressed under higher compression force without compromising critical tablet properties.



Figure 11. The compression profiles of the original formula, the optimized formula, and the formula with $20 \mathrm{wt} \% \mathrm{PH}-102$.

In production, we have observed that a formula had been successfully manufactured for many years but started to show chipping and capping. A plausible reason could be that the particle sizes of raw materials shifted lower and the formula needs to be compressed at a higher force in order to reach the target hardness. This hypothesis is based on the evaluation of sieved blends shown Figure 8, where the particle size of the whole formula blend became smaller and the formula became less compressible. A robust formula of a wide operating range can be compressed at a higher compression force without chipping or capping, as demonstrated in Figure 11. However, if the formula is not robust, capping and chipping may be observed at very high compression forces. The solution is to design a formula resilient to raw material variations, environment, storage conditions, and equipment setup. Optimizing key ingredient concentrations in the formula with DOE and selecting suitable binder materials are critical.

The results from Figures 8 and 9 seem to contradict each other in term of how particle size impact on compressibility. Figure 8 demonstrates that a formula with smaller particle size is less compressible; however, after smaller particle size binder particles were introduced into the formula, the formula becomes very compressible, as shown in Figure 9. Typically tablet formulas contain a distribution of particle size and the particle size of different functional materials may impact compressibility in different ways. A microscopic physical mechanism of how powder particles are packed and compressed is therefore proposed in the next section to explain the data.

\subsection{Physical Mechanism of Powder Compressions}

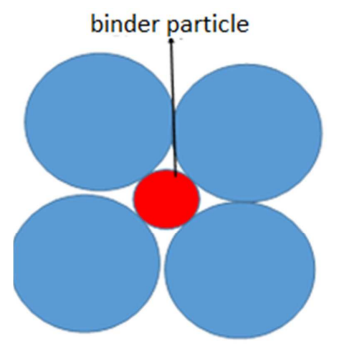

a. Closely match to interstitial space

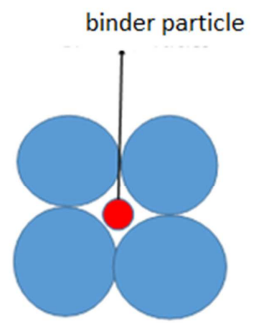

b. Smaller than interstitial space

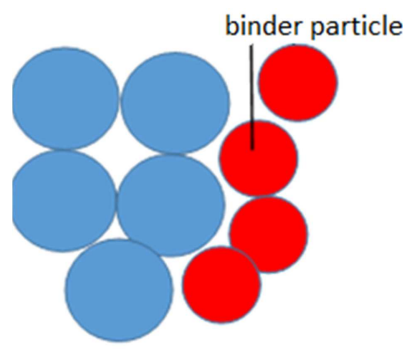

c. Larger than interstitial space

Figure 12. Physical hypothesis based on particle sizes: the interstitial space formed by the particles in the formula is critical for selecting the binder ingredient of matched particle sizes. The interstitial space created by ingredient particles other than binder: a), is comparable to the particle sizes of the binder; b), is much larger than the particle sizes of the binder; $c$ ), is much smaller than the particle sizes of the binder and all the binder particles sit outside of the interstitial spaces.

The seemingly contradictory experimental observations mentioned in prior section could be explained with how the particles in a formula are bound together. Figure 12 shows a possible microscopic mechanism behind this phenomenon. Depending on the relative particle size of the binder particles to that of the remaining particles, three particle packing scenarios could occur in term of the interstitial space created by the ingredient particles other than binder's: a), the interstitial space is comparable to the particle size of the binder; b), the interstitial space is much larger than the particle size of the binder; c), the interstitial space is much smaller than the particle size of the binder. The binder particles function as a "glue" that holds different particles together in the tablet. If there are sufficient binder particles and the binder particles are strong, tablets with a very low friability would be produced; the defects like chipping, capping, and dusting would be unlikely to occur. For a robust formula where particle size of raw materials is optimized, the binder particles can sit perfectly in the interstitial spaces created by other ingredients and the particle size of the binder 
is comparable to that of the interstitial space as shown in Figure 12a. An efficient compression process is thus expected. When a compression force is applied to the powder bed, the binder particles that are sitting in the interstitial spaces will be immediately compressed and deform to bind the surrounding particles together. If the binder particle size is not well matched to the size of the interstitial spaces, the applied energy is to crush or deform the surrounding particles first before acting on binders, this could require a higher compression force to reach the same compressibility. The binder ingredients are typically soft materials made from polymers like MCC or other organic soft materials. Unlike inorganic particles, such as minerals in a typical nutraceutical formula, these binder particles can be compressed easily under a small compression force, achieving the desired hardness with much lower compression force [3]. In an ideal formula, the particle size of the binder matches that of the remaining particles forming the interstitial spaces, and each interstitial space or cavity formed by other particles should contain at least one binder particle to form bound structures.

This physical picture could explain why the formula containing the small binder particle, like UF-711 and KG-1000 shown in Figure 9, shows much better compressibility. The needle-shaped particles, like KG-1000, have limited contact points with other particles or cannot sit perfectly inside the interstitial spaces, which could explain why the formula with KG-1000 is less compressible than that with UF-711. In contrast, if the particle size of the binder is much smaller than the size of the interstitial spaces as shown in Figure 12b, the surrounding particles need to be displaced and/or compressed before the binder particles. If the surrounding particles are hard materials like minerals, relatively higher compression force is needed. Of course, if the surrounding particles are hard but not very abrasive, the compression force can be easily transferred to the binder particles without any frictional loss, and there should be minimal difference between the cases shown in Figures 12a and $12 \mathrm{~b}$. However, if the surrounding particles are brittle or plastic, the applied compression force cannot be immediately transferred to the binder particles, extra compression force is required to compress the surrounding particles. The worst scenario is the case when the binder particles are much larger than the size of the interstitial spaces and the binder particles are outside of the interstitial spaces due to the size-induced segregation phenomena, as depicted in Figure 12c. In such a case, binder particles have very limited contacts with other particles and cannot function as a "glue" to hold the particles together. Binder particles may segregate from other ingredients and cannot be evenly distributed in the powder system. In this case, the binder particles bind together strongly and other particles are only weakly associated with each other. The tablet will be dusty and have chipping and capping issues. When the particle size of the whole formula is smaller, as demonstrated in Figure 8, the particles cannot be compressed under similar compression force with similar displacement. In this instance, strong compression force is needed in order to crush the particles for them to bind together. In addition, the binder particle must be small enough to fit the interstitial spaces and should become even smaller when other particles are small. The larger binder particle relative to the remaining particles would create a situation as shown in Figure 12c, leading to poor quality tablets.

The key is to find the right particle size of the binder used in the formula relative to the remaining particles. The particle size of the powder blend should be characterized for determining the minimum particle size of the binder required in the formula, under certain assumptions on particle packing structures like cubic structure, random loose and random close packing structures [13]. The interstitial space size can be estimated with the method proposed by Hao and Riman [14]. The concentration of binder particles is also critical as demonstrated in Figures 10 and 11 and explained in the literature [15]. If a continuous binder particle network after compression can be formed three dimensionally inside tablets, strong tablets without capping and chipping would be expected.

\section{Summary and Conclusion}

We have demonstrated that DOE is a useful tool to identify components with the greatest impact on critical product attributes and to refine the concentrations of those components to achieve an optimal formula. We start with typical nutraceutical tablet formulas containing a flow agent, disintegrant, lubricant, binder and active ingredients. A robust formulation must withstand typical variations in raw materials and process. Developing a formula with the broadest possible design space enables the potential robustness to such variations. In the formulas examined in this article, granular mannitol was identified as the most critical component and the level was optimized to improve compressibility. The formula was further improved with the addition of MCC as a binder, expanding the potential operating ranges. The scalability of the results was demonstrated, confirming that less resource-intensive benchtop DOE experiments can be effectively translated to more commercially scalable trials.

What we have found is that the particle size of the binder and the reminder particles is critical for a successful compression profile. A microscopic physical mechanism of powders under compression is proposed to explain the experimental observations related to the impact of particle size on compressibility. In general, binder particles smaller than other ingredients in the formula will more likely fit into the interstitial spaces created by other particles and enhance the compression profile. Designing formulas resilient to raw materials and equipment variations will allow for the formulas to be produced at a wider operating range. Specifically, the concentration of each key ingredient must be optimized using DOE method and the binder particle size must match that of the remaining particles. This optimization process will ensure tablet product free of chipping and capping issues. 


\section{Acknowledgements}

We thank Mike Naddell, Raul Lopez, and Barbara Montano for assisting with the experiments and Lana L. Woshnak and Susan Hazels Mitmesser for oversight of the experiments \& editing the manuscript.

\section{References}

[1] K. Zaheer and P. Langguth, "Designing robust immediate release tablet formulations avoiding food effects for BCS class 3 drugs", 2019, Eur. J. Pharm. Biopharm., 139, pp 177-185.

[2] K. Ofori-Kwakye, F. Osei-Yeboah, and S. L. Kipo, "Formulation and quality evaluation of two conventional release tablet formulations", 2010, Intern. J. Pharm. Sci. Rev. Res., 4 (1), pp 94-99.

[3] M. Capece, Zhonghui Huang, and Rajesh Dave, "Insight Into a Novel Strategy for the Design of Tablet Formulations Intended for Direct Compression”, 2017, J. Pharm. Sci, 106 (6), pp 1608-1617.

[4] S. Chattoraj, P. Daugherity, T. McDermott, A. Olsofsky, W. J. Roth, and M. Tobyn, "Sticking and Picking in Pharmaceutical Tablet Compression: An IQ Consortium Review", 2018, J. Pharm. Sci., 107 (9), pp 2267-2282.

[5] J. Tao, S. Robertson-Lavalle, P. Pandey, and S. Badawy, "Understanding the Delamination Risk of a Trilayer Tablet Using Minipiloting Tools", 2017, J. Pharm. Sci., 106 (11), pp 3346-3352.

[6] A. Skelbæk-Pedersen, T. Vilhelmsen, V. Wallaert, and J. Rantanen, "Quantification of Fragmentation of Pharmaceutical
Materials After Tableting", 2018, J. Pharm. Sci., 108 (3), pp $1246-1253$.

[7] C. K. Tye, C. Sun, G. E. Amidon, "Evaluation of the effects of tableting speed on the relationships between compaction pressure, tablet tensile strength, and tablet solid fraction", 2005, J. Pharm. Sci., 94 (3), pp 465-472.

[8] H. Goh, P. Wan, S. Heng, C. V. Liew, "The Effects of Feed Frame Parameters and Turret Speed on Mini-Tablet Compression”, 2018, J. Pharm. Sci., 108 (3), pp 1161-1171.

[9] S. Abdel-Hamid, F. Alshihabi, G. Betz, "Investigating the effect of particle size and shape on high speed tableting through radial die-wall pressure monitoring", 2011, Int. J. Pharm., 413 (1-2), pp 29-35.

[10] D. C. Montgomery, Design and Analysis of Experiments, $8^{\text {th }}$ ed., John Wiley \& Sons, Inc, 2013.

[11] Jinjiang Li and Yongmei Wu, "Lubricants in Pharmaceutical Solid Dosage Forms”, Lubricants, 2014, 2 (1), pp 21-43.

[12] The data are from Asahi Kasei website, https://www.ceolus.com/en/ceolus_basic.html

[13] Dennis R. Dinger and James E. Funk, Particle-Packing Phenomena and Their Application in Materials Processing, MRS Bulletin 1997, 22 (12), pp. 19-23.

[14] T. Hao, and R. Riman, "Calculation of interparticle spacing in colloidal systems" Journal of Colloid And Interface Science, 2006, $297 \quad$ (1), $\quad$ pp 374-377, https://doi.org/10.1016/j.jcis.2004.10.014Y.

[15] C. Calvin Sun, "Microstructure of Tablet-Pharmaceutical Significance, Assessment, and Engineering", Pharmaceutical Research, 2017, 34 (5), pp 918-928. 